\title{
HELICITY-FLIP OFF-FORWARD PARTON DISTRIBUTIONS OF THE NUCLEON
}

\author{
Pervez Hoodbhoy* and Xiangdong Ji \\ Department of Physics \\ University of Maryland \\ College Park, Maryland 20742 \\ (U. of MD PP\#98-077 DE/ER/40762-142 January 1998)
}

\begin{abstract}
We identify quark and gluon helicity-flip distributions defined between nucleon states of unequal momenta. The evolution of these distributions with change of renormalization scale is calculated in the leading-logarithmic approximation. The helicity-flip gluon distributions do not mix with any quark distribution and are thus a unique signature of gluons in the nucleon. Their contribution to the generalized virtual Compton process is obtained both in the form of a factorization theorem and an operator product expansion. In deeply virtual Compton scattering, they can be probed through distinct angular dependence of the cross section.
\end{abstract}

*Fulbright scholar, on leave from Department of Physics, Quaid-e-Azam University, Islamabad 45320, Pakistan. 


\section{INTRODUCTION}

From the point of view of quantum field theory a nucleon is fully described only if one knows the matrix elements of all possible quark and gluon operators involving the nucleon state. Nevertheless, progress is possible provided one can obtain the matrix element of operators with a clear physical interpretation. Twist-two operators give the leading contribution in appropriate hard processes, are relatively simple, and are more accessible to experimental measurement. The states between which these operators are sandwiched may be of equal or unequal momenta; the former situation is familiar from well-investigated processes like deeply inelastic scattering. The latter has also been investigated over the years [1]. However their importance has been understood only recently. For instance, knowing certain off-forward twist-two matrix elements allows for extraction of the quark and gluon orbital and spin contributions to the nucleon spin [2]. The class of parton distributions, known as off-forward (off-diagonal, non-forward) parton distributions defined from these off-forward matrix elements has generated considerable contemporary interest [2 11, 15, 13]. In simple physical terms, a parton distribution, whether forward or off-forward, arises from removal of the parton from the nucleon by a hard probe and its subsequent return to form the nucleon ground state further along the light-cone, or a similar process.

The class of twist-two operators which depends on parton helicity change shall be the subject of this paper. It is now well known [14,16, 17] that there is one forward chirally odd twist-two proton structure function, known as $h_{1}\left(x, Q^{2}\right)$, measurable in, for example, the Drell-Yan process. It is defined as the light-cone correlation of quark fields weighted by $\sigma^{\mu \nu}$,

$$
\int \frac{d \lambda}{2 \pi} e^{i \lambda x}\left\langle P S^{\prime}\left|\bar{\psi}\left(-\frac{1}{2} \lambda n\right) \sigma^{\mu \nu} \psi\left(\frac{1}{2} \lambda n\right)\right| P S\right\rangle=h_{1}(x) \bar{U}\left(P S^{\prime}\right) \sigma^{\mu \nu} U(P S)+\ldots
$$

In a helicity basis, wherein spins are measured along the particle's momentum and $\Sigma_{\|}$is diagonal, $S$ and $S^{\prime}$ differ by one unit of angular momentum. Since chirality and helicity coincide for massless quarks, $h_{1}$ is a chiral-odd quantity. To give it a probability interpretation requires using a transversity basis wherein $\Sigma_{\perp} \gamma_{0}$ is diagonal. This gives $h_{1}$ the necessary probabilistic interpretation (for states of equal momenta): it is the probability to find a quark polarized along the transverse polarization of the nucleon minus the probability to find the quark polarized in the opposite direction. There is no gluonic helicity-flip distribution for obvious reason - a transverse gluon flipping its helicity leads to a change of two

units of angular momentum and angular momentum conservation forbids this for a spin- $\frac{1}{2}$ hadron. For hadrons with spin $\geq 1$ there is no such restriction and some years ago Jaffe and Manohar [18] identified a leading twist gluonic structure function $\Delta\left(x, Q^{2}\right)$ which can be measured from a transversely polarized target like the deuteron.

New Lorentz structures emerge if one allows for off-forward matrix elements, leading to generalizations of the above mentioned helicity changing structure functions. Recently, Collins et al. have suggested measuring the helicity-flip quark distributions in vector meson production [19,20]. The evolution equation for these distributions has been derived by Belitsky and Müller [1]. Diehl et al. 21] have noticed that the distribution in angle between the lepton and hadron planes in deeply virtual Compton scattering (DVCS) contains valuable information about the helicity structure of the nucleon-photon amplitudes. They point out that photon helicity flip is possible even with a spin- $\frac{1}{2}$ target because gluons in the offforward scattering can transfer two units of angular momentum. This, of course, requires 
the existence of the gluon helicity-flip distributions in the nucleon. Indeed, for off-forward matrix elements one does not need a state of spin $\geq 1$ to accomodate gluon helicity flip.

This paper is intended to present a comprehensive study of leading-twist helicity-flip off-forward distributions in the nucleon. A systematic counting suggests that there are four such distributions: two related to gluon helicity flip and the other two to quark helicity flip. In each case, a distribution can be defined depending on whether the nucleon's helicity is flipped or not. We derive the leading-logarithmic evolution of these distributions, although in the quark case, the result was already obtained by Belitsky and Müller [15]. In the forward limit, the evolution of the quark distributions reduces to that of $h_{1}(x)$ as calculated by Artru and Mekhfi [16]. The evolution for the gluon distributions reduces to that of $\Delta(x)$, which has not appeared in the literature before. Note that the quark helicity-flip distributions do not mix with any gluon ones, and vice versa. This is quite significant because, for the first time, we have a parton distribution that can serve as a unique signature of the gluons inside the nucleon: gluonic effects cannot be mocked up by any kind of constituent quarks, and they cannot be evolved away by recklessly evolving down the momentum scale!

We study measurement of the helicity-flip gluon distributions in general two-photon process. The photon helicity-flip Compton amplitude is calculated in terms of the gluon helicity-flip distributions. In the forward limit, we recover the result obtained in 18 . However, our result is more general. In the language of operator product expansion, we obtain the leading-order coefficient functions of a class of gluon operators with total derivatives. According to [21], the helicity-flip gluon distributions generate distinct angular distributions in the DVCS cross section.

The presentation of the paper is as follows. In Section II, we enumerate the independent helicity amplitudes for the quark-nucleon and gluon-nucleon sub-processes. Subsequently new helicity changing distribution functions are motivated and defined. In Section III the leading logarithmic evolution of these functions is studied. Section IV contains a calculation of the Compton amplitude for photon helicity flip scattering. This amplitude vanishes at the tree level and requires at least one quark box (plus permutations) to be non-zero. Section $\mathrm{V}$ presents the DVCS cross section that depends on the gluon helicity-flip distributions. We conclude the paper in Sec. VI.

\section{HELICITY-FLIP PARTON DISTRIBUTIONS: COUNTING AND DEFINITIONS}

We shall, in this section, enumerate the complete set of off-forward quark and gluon distributions at the twist-two level. Helicity-flip ones will emerge through the counting and will be the focus of this paper. As usual, $p^{\mu}$ and $n^{\mu}$ are two light-like vectors with $p^{2}=n^{2}=0$ and $p \cdot n=1$. The momenta and spins of the initial and final nucleons are, respectively, $P, S$ and $P^{\prime}, S^{\prime}$. The momentum transfer $\Delta^{\mu}=P^{\prime \mu}-P^{\mu}$ has both transverse and longitudinal components. It is convenient to define a special system of coordinates wherein $\bar{P}^{\mu}=\left(P^{\prime}+P\right)^{\mu} / 2$ is collinear and in the $z$ direction,

$$
\begin{aligned}
& \bar{P}^{\mu}=p^{\mu}+\left(\bar{M}^{2} / 2\right) n^{\mu}, \\
& \Delta^{\mu}=-2 \xi\left(p^{\mu}-\left(\bar{M}^{2} / 2\right) n^{\mu}\right)+\Delta_{\perp}^{\mu}, \\
& \bar{M}^{2}=M^{2}-\Delta^{2} / 4 .
\end{aligned}
$$


The initial nucleon and parton have longitudinal momentum fractions $1+\xi$ and $x+\xi$, respectively.

From dimensional reasoning, the leading order contribution to a given hard process must involve the minimum number of independent parton fields which, for QCD quantized on the light cone, is two ( $\psi_{+}$for fermions and $A_{\perp}$ for gluons). Therefore, one need consider only the matrix elements of bilinear operators at two different points on the light-cone. In the kinematic region $x>\xi$, one has the simple interpretation that the first operator extracts a certain type of parton from the nucleon and the second replaces it further along the light-cone. Let $H, H^{\prime}$ denote the respective helicities of the initial and final nucleon and $h, h^{\prime}$ the helicities of the parton extracted and replaced. The helicity amplitude $\mathcal{A}_{H h, H^{\prime} h^{\prime}}$ must obey $\mathcal{A}_{H^{\prime} h^{\prime}, H h}=\mathcal{A}_{H h, H^{\prime} h^{\prime}}$ (time-reversal invariance), and $\mathcal{A}_{-H-h,-H^{\prime}-h^{\prime}}=\mathcal{A}_{H h, H^{\prime} h^{\prime}}$ (parity invariance). For a purely collinear process there is no preferred transverse direction; rotational invariance around the collinear axis requires helicity to be conserved, $H+h^{\prime}=$ $H^{\prime}+h$. However, non-zero transverse momentum of the scattered nucleon or parton means that, while the total angular momentum will of course be conserved, helicity conservation will not necessarily hold. The difference is, of course, absorbed by the orbital motion of the scattered pair.

For quarks it is readily seen that a set of independent amplitudes is provided by the following: $\mathcal{A}_{\frac{1}{2} \frac{1}{2}, \frac{1}{2} \frac{1}{2}}, \mathcal{A}_{\frac{1}{2}-\frac{1}{2}, \frac{1}{2}-\frac{1}{2}}, \mathcal{A}_{\frac{1}{2} \frac{1}{2},-\frac{1}{2}-\frac{1}{2}} \mathcal{A}_{\frac{1}{2} \frac{1}{2}, \frac{1}{2}-\frac{1}{2}}, \mathcal{A}_{\frac{1}{2} \frac{1}{2},-\frac{1}{2} \frac{1}{2}}$, and $\mathcal{A}_{\frac{1}{2}-\frac{1}{2},-\frac{1}{2} \frac{1}{2}}$. The familiar distributions $f_{1}\left(x, Q^{2}\right), g_{1}\left(x, Q^{2}\right)$, and $h_{1}\left(x, Q^{2}\right)$ are linear combinations of the first three in the forward limit [17]. One unit of orbital angular momentum, made available by one power of $\Delta_{\perp}$, allows for three additional amplitudes. A complete set of off-forward leading-twist quark distributions is given below:

$$
\begin{aligned}
\int \frac{d \lambda}{2 \pi} e^{i \lambda x}\left\langle P^{\prime} S^{\prime}\left|\bar{\psi}_{q}\left(-\frac{1}{2} \lambda n\right) \gamma^{\mu} \psi_{q}\left(\frac{1}{2} \lambda n\right)\right| P S\right\rangle= & H_{q}(x, \xi) \bar{U}\left(P^{\prime} S^{\prime}\right) \gamma^{\mu} U(P S) \\
& +E_{q}(x, \xi) \bar{U}\left(P^{\prime} S^{\prime}\right) \frac{i \sigma^{\mu \nu} \Delta_{\nu}}{2 M} U(P S)+\ldots \\
\int \frac{d \lambda}{2 \pi} e^{i \lambda x}\left\langle P^{\prime} S^{\prime}\left|\bar{\psi}_{q}\left(-\frac{1}{2} \lambda n\right) \gamma^{\mu} \gamma_{5} \psi_{q}\left(\frac{1}{2} \lambda n\right)\right| P S\right\rangle= & \tilde{H}_{q}(x, \xi) \bar{U}\left(P^{\prime} S^{\prime}\right) \gamma^{\mu} \gamma_{5} U(P S) \\
& +\tilde{E}_{q}(x, \xi) \bar{U}\left(P^{\prime} S^{\prime}\right) \frac{\gamma_{5} \Delta^{\mu}}{2 M} U(P S)+\ldots \\
\int \frac{d \lambda}{2 \pi} e^{i \lambda x}\left\langle P^{\prime} S^{\prime}\left|\bar{\psi}_{q}\left(-\frac{1}{2} \lambda n\right) \sigma^{\mu \nu} \psi_{q}\left(\frac{1}{2} \lambda n\right)\right| P S\right\rangle= & H_{T q}(x, \xi) \bar{U}\left(P^{\prime} S^{\prime}\right) \sigma^{\mu \nu} U(P S) \\
& +E_{T q}(x, \xi) \bar{U}\left(P^{\prime} S^{\prime}\right) \frac{\gamma^{[\mu} i \Delta^{\nu]}}{M} U(P S)+\ldots
\end{aligned}
$$

where $[\mu \nu]$ means antisymmetrization of the two indices and the ellipses denote higher twist structures which are outside the scope of the present discussion. The dependence of each distribution upon $t=\Delta^{2}$ and $Q^{2}$ is implicit. In each equation, the first term represents an amplitude that survives the forward limit and the second term an amplitude that decouples (but does not vanish) in the forward limit. The definitions of $H_{q}, E_{q}, \tilde{H}_{q}$, and $\tilde{E}_{q}$ are from Ref. [2]. The quark helicity-flip distributions $H_{T q}$ and $E_{T q}$ are new; and they can be selected from the third equation by taking $\mu=+$ and $\nu=\perp$. The above definitions complete the identification of all twist-two quark distributions.

A few additional comments about the definition in Eq. (3) are in order. First, for brevity we have not explicitly shown the gauge link between the quark fields. This link is always 
present, except in the light-like gauge $A^{+}=0$. Second, by using time-reversal symmetry, all the distributions are seen to be real. Third, from taking the complex conjugate of the above equations, it follows that all the distributions are even functions of $\xi$. Finally, we can add a time-ordering between the two fields without changing the content. This follows from,

$$
T \psi_{+}^{\dagger}(0) \psi_{+}(\lambda n)=\psi_{+}^{\dagger}(0) \psi_{+}(\lambda n)-\theta\left(\lambda n^{0}\right)\left\{\psi_{+}^{\dagger}(0), \psi_{+}(\lambda n)\right\} .
$$

The second term is just a constant because it is an anticommutator of the independent (or good) components of the Dirac field separated along the light cone. Obviously, the constant does not contribute to the matrix elements. More elaborate but essentially equivalent proofs can be found in the literature [22,23].

We now turn to the gluon distributions. Only transverse gluons need be considered here because longitudinal ones are either dependent or gauge degrees of freedom, which lead to either higher twist distributions or gauge links. An independent set of nucleon gluon amplitudes is: $\mathcal{A}_{\frac{1}{2} 1, \frac{1}{2} 1}, \mathcal{A}_{\frac{1}{2}-1, \frac{1}{2}-1}, \mathcal{A}_{\frac{1}{2} 1,-\frac{1}{2}-1} \mathcal{A}_{\frac{1}{2} 1, \frac{1}{2}-1}, \mathcal{A}_{\frac{1}{2} 1,-\frac{1}{2} 1}$, and $\mathcal{A}_{\frac{1}{2}-1,-\frac{1}{2} 1}$. The familiar distributions $G\left(x, Q^{2}\right)$ and $\Delta G\left(x, Q^{2}\right)$ come from the forward limit of the first two amplitudes. There is no equivalent of $h_{1}$ for gluons since it is impossible for a nucleon to spin-flip by two units. All off-forward twist-two gluon distributions are defined below:

$$
\begin{aligned}
& \frac{1}{x} \int \frac{d \lambda}{2 \pi} e^{i \lambda x}\left\langle P^{\prime} S^{\prime}\left|F^{(\mu \alpha}\left(-\frac{\lambda}{2} n\right) F_{\alpha}{ }^{\nu)}\left(\frac{\lambda}{2} n\right)\right| P S\right\rangle \\
& \quad=H_{g}(x, \xi) \bar{U}\left(P^{\prime} S^{\prime}\right) \bar{P}^{(\mu} \gamma^{\nu)} U(P S)+E_{g}(x, \xi) \bar{U}\left(P^{\prime} S^{\prime}\right) \frac{\bar{P}^{(\mu} i \sigma^{\nu) \alpha} \Delta_{\alpha}}{2 M} U(P S)+\ldots \\
& \frac{1}{x} \int \frac{d \lambda}{2 \pi} e^{i \lambda x}\left\langle P^{\prime} S^{\prime}\left|F^{(\mu \alpha}\left(-\frac{\lambda}{2} n\right) i \tilde{F}_{\alpha}{ }^{\nu)}\left(\frac{\lambda}{2} n\right)\right| P S\right\rangle \\
& =\tilde{H}_{g}(x, \xi) \bar{U}\left(P^{\prime} S^{\prime}\right) \bar{P}^{(\mu} \gamma^{\nu)} \gamma_{5} U(P S)+\tilde{E}_{g}(x, \xi) \bar{U}\left(P^{\prime} S^{\prime}\right) \frac{\gamma_{5} \bar{P}^{(\mu} \Delta^{\nu)}}{2 M} U(P S)+\ldots \\
& \frac{1}{x} \int \frac{d \lambda}{2 \pi} e^{i \lambda x}\left\langle P^{\prime} S^{\prime}\left|F^{(\mu \alpha}\left(-\frac{\lambda}{2} n\right) F^{\nu \beta)}\left(\frac{\lambda}{2} n\right)\right| P S\right\rangle \\
& =H_{T g}(x, \xi) \bar{U}\left(P^{\prime} S^{\prime}\right) \frac{\bar{P}^{([\mu} i \Delta^{\alpha]} \sigma^{\nu \beta)}}{M} U(P S) \\
& \quad+\quad E_{T g}(x, \xi) \bar{U}\left(P^{\prime} S^{\prime}\right) \frac{\bar{P}^{([\mu} \Delta^{\alpha]}}{M} \frac{\gamma^{[\nu} \Delta^{\beta])}}{M} U(P S)+\ldots
\end{aligned}
$$

Here in the first two equations, $(\mu \nu)$ means symmetrization of the two indices and removal of the trace, and in the third equation $[\mu \alpha]$ and $[\nu \beta]$ are antisymmetric pairs and $(\cdots)$ signifies symmetrization of the two and removal of the trace. These operations are essential since the product of operators must transform as irreducible representations of the Lorentz group.

The distributions $H_{g}, E_{g}, \tilde{H}_{g}$, and $\tilde{E}_{g}$ have been introduced before [3, 4]. Their evolutions and mixing with quark distributions have also been worked out. They play an important roles in electro-meson production in the small $x$ region [9,24]. The helicity-flip distributions $E_{T g}$ and $H_{T g}$ are new. They can be selected from Eq. (5) by taking $\mu=\nu=+$ and $\alpha, \beta=\perp$. The angular momentum conservation requires presence of one unit of angular momentum $\left(\Delta_{T}\right)$ when the nucleon helicity is flipped $\left(H_{T g}\right)$ and two units $\left(\Delta_{T} \Delta_{T}\right)$ when it is not $\left(E_{T g}\right)$. Hence both decouple from the matrix elements in the forward limit although the distributions themselves do not vanish. 
In a sense, the gluon helicity-flip distributions are the "cleanest" among the class of gluon distributions since they are forbidden to mix with quark distributions by angular momentum conservation. This, in fact, was why Jaffe and Manohar [18] had proposed using the $\Delta\left(x, Q^{2}\right)$ distribution as a probe of "exotic gluons" in a spin $J \geq 1$ nucleus. Nuclear binding or pions would not contribute. However there, unlike here, there is a strong suppression on the magnitude of the distribution due to the small size of the nuclear interaction relative to a typical hadronic mass scale because "exotic gluons" can only be generated by the nuclear interaction (for an estimate of $\Delta\left(x, Q^{2}\right)$ see Ref.( [25]) ). In the nucleon, the off-forward helicity-flip gluon distributions can be as large as other gluon distributions for a reasonable size of $x$. It is interesting to note that the $\mathrm{n}=2$ moment of the gluon distributions above can be directly expressed in terms of the matrix elements of the colour electric and magnetic fields in the nucleon.

\section{EVOLUTION OF HELICITY-FLIP DISTRIBUTIONS}

A parton distribution is necessarily defined at a given distance or momentum scale because of ultraviolet divergences. Physically, the scale can be related to the kinematic variables of the particular hard process under consideration. In this section we study, using momentum space Feynman diagrams, the leading-logarithmic evolution of the helicity-flip parton distributions defined in the previous section. While the method is straightforward in principle, there are important subtleties related to the gauge dependence of the calculation and end-point singularities. In principle any gauge choice is permissible but the light-cone gauge is naturally preferred for several reasons: the fewest number of diagrams need be calculated, path-ordered exponentials are absent, the light-cone dynamics of partons is transparent, and ghosts are absent. However, there is a price to be paid because the lightcone gluon propagator, when imbedded in a loop, leads to singularities in the end-points of integrals whose interpretation is ambiguous. This is sufficient reason to do the (longer) calculation in a covariant gauge. Therefore, in the following, we shall use the Feynman gauge and treat separately the quark and gluon helicity-flip distributions. At leading-logarithmic order, any ultraviolet regulator is as good as any other; so we impose an ultraviolet cut-off on the momentum integrations.

\section{A. Evolution of the Quark Distributions $E_{T}$ and $H_{T}$}

The result in this section has been obtained before in Ref. [11]. Here for completeness we present our calculation in a different form.

From the definitions of the quark distributions $H_{T q}$ and $E_{T q}$ in Eq.(3), it is clear that the two will evolve identically with the leading component of the operator $\bar{\psi} \sigma^{\mu \nu} \psi$. Selecting only the leading twist part, it is therefore convenient to define,

$$
\mathcal{F}\left(x, \xi, Q^{2}\right)=n_{\mu} e_{\nu} \int \frac{d \lambda}{2 \pi} e^{i \lambda x}\left\langle P^{\prime} S^{\prime}\left|\bar{\psi}\left(-\frac{1}{2} \lambda n\right) \sigma^{\mu \nu} e^{-i g \int_{\lambda / 2}^{-\lambda / 2} d \alpha n \cdot A(\alpha n)} \psi\left(\frac{1}{2} \lambda n\right)\right| P S\right\rangle .
$$

where $e^{\mu}$ is a unit vector in a transverse direction. The path-ordered integral has been

reinstated in the above. The renormalization scale $Q^{2}$ is the cut-off for the momentum 
components of the fields. $\mathcal{F}\left(x, \xi, Q^{2}\right)$ can be diagrammatically represented as in Fig.1a. Now imagine a slight increase of $Q^{2}$ to $Q^{2}+\delta Q^{2}$, revealing a deeper level of hadronic substructure. Additional diagrams contributing to $\mathcal{F}\left(x, \xi, Q^{2}+\delta Q^{2}\right)$ are shown in Figs. 1b-1e. 


\section{FIGURES}

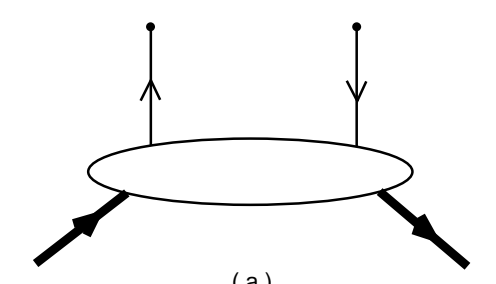

( a )

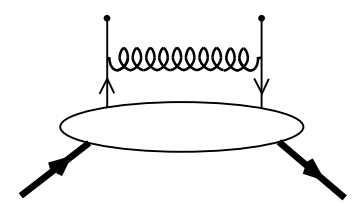

(b)

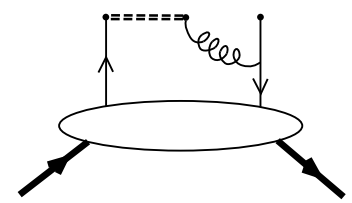

(c)

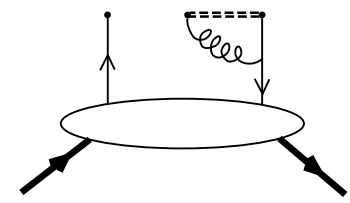

(d)

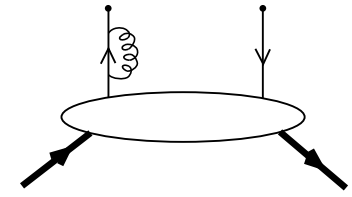

(e)

FIG. 1. Feynman diagrams for evolution of quark helicity-flip parton distributions.

Feynman-like rules for these diagrams can be derived in a rather straightforward way: the product of bare Heisenberg fields in Eq.(16) can be brought under the time-ordering symbol and a perturbation expansion follows from expanding out the exponential containing the QCD interaction terms $\exp \left(i \int \mathcal{L}_{\text {int }}\right)$ as well as expanding the path-ordered exponential. Subsequently a collinear expansion is made: the lines entering or leaving the hadron blob are always collinear, reflecting the fact that the internal relative momenta of partons in the hadron are much less than the momentum of the hard probe. The transverse momenta of the other lines is bounded by $Q^{2}$.

The Feynman expressions for fig.1c and 1d are,

$$
\begin{aligned}
\delta \mathcal{F}_{c}\left(x, \xi, Q^{2}\right)= & \frac{1}{2} n_{[\mu} e_{\nu]} \int d y \int \frac{d^{4} k}{(2 \pi)^{4}} \delta(x-k \cdot n) \frac{i}{(q-k) \cdot n+i \epsilon} i D_{\alpha \beta}(q-k) \\
& \times \operatorname{Tr}\left[\frac{1}{8} \sigma^{\mu \nu}\left(-i g t^{a} n^{\alpha}\right) \sigma^{\rho \lambda}\left(-i g t^{a} \gamma^{\beta}\right) i S_{F}\left(k+\frac{1}{2} \Delta\right)\right] p_{[\rho} e_{\lambda]} \mathcal{F}\left(y, \xi, Q^{2}\right), \\
\delta \mathcal{F}_{d}\left(x, \xi, Q^{2}\right)= & \frac{1}{2} n_{[\mu} e_{\nu]} \int d y \int \frac{d^{4} k}{(2 \pi)^{4}} \delta(x-y) \frac{i}{(k-q) \cdot n+i \epsilon} i D_{\alpha \beta}(q-k) \\
& \times \operatorname{Tr}\left[\frac{1}{8} \sigma^{\mu \nu}\left(-i g t^{a} n^{\alpha}\right) \sigma^{\rho \lambda}\left(-i g t^{a} \gamma^{\beta}\right) i S_{F}\left(k+\frac{1}{2} \Delta\right)\right] p_{[\rho} e_{\lambda]} \mathcal{F}\left(y, \xi, Q^{2}\right) .
\end{aligned}
$$

In the first of the above two equations, $q^{\mu}=y p^{\mu}$ and in the second, $q^{\mu}=x p^{\mu}$. Two other diagrams are obtained by reflection and yield identical expressions under $\xi \rightarrow-\xi$. Finally, the quark self-energy diagram in fig.1e (and its reflection), together with the wavefunction renormalization, yield the combined expression below after calculation,

$$
\begin{aligned}
\frac{D_{Q} \mathcal{F}\left(x, \xi, Q^{2}\right)}{D \ln Q^{2}}= & \frac{\alpha_{s}\left(Q^{2}\right)}{2 \pi} C_{F}\left[\theta(x-\xi) \int_{x}^{1} \frac{d y}{y} \frac{x-\xi}{y-\xi}+\theta(x+\xi) \int_{x}^{1} \frac{d y}{y} \frac{x+\xi}{y+\xi}\right. \\
& \left.-\theta(\xi-x) \int_{-1}^{x} \frac{d y}{y} \frac{x-\xi}{y-\xi}-\theta(-\xi-x) \int_{-1}^{x} \frac{d y}{y} \frac{x+\xi}{y+\xi}\right] \frac{\mathcal{F}\left(y, \xi, Q^{2}\right)}{y-x+i \epsilon},
\end{aligned}
$$

where, 


$$
\frac{D_{Q}}{D \ln Q^{2}}=\frac{d}{d \ln Q^{2}}-\frac{\alpha_{s}\left(Q^{2}\right)}{2 \pi} C_{F}\left[\frac{3}{2}+\int_{\xi}^{x} \frac{d y}{y-x-i \epsilon}+\int_{-\xi}^{x} \frac{d y}{y-x-i \epsilon}\right] .
$$

The above result agrees with that obtained by Belitsky and Müller.

It is useful to take moments of $\mathcal{F}\left(x, \xi, Q^{2}\right)$. Define,

$$
\mathcal{F}_{n}\left(\xi, Q^{2}\right)=\int_{-1}^{1} d x x^{n-1} \mathcal{F}\left(x, \xi, Q^{2}\right) \quad(n \geq 2),
$$

where $n=$ even (odd) moments are charge conjugation even (odd). Then, a calculation leads to the following evolution equation for the moments,

$$
\frac{d \mathcal{F}_{n}}{d \ln Q^{2}}=\frac{\alpha_{s}\left(Q^{2}\right)}{2 \pi} C_{F}\left[\left(\frac{3}{2}-2 S(n)\right) \mathcal{F}_{n}+2 \sum_{i=1,2, . .}^{\left[\frac{n-1}{2}\right]}\left(\frac{1}{2 i}-\frac{1}{n}\right)(2 \xi)^{2 i} \mathcal{F}_{n-2 i}\right],
$$

where,

$$
S(n)=\sum_{i=1}^{n} \frac{1}{i}
$$

We shall now interpret the evolution equation in terms of operator mixing. To this end, define,

$$
\mathcal{O}_{n, 2 i}^{\mu_{1} \cdots \mu_{n} \nu}(x)=i \partial^{\mu_{1}} \cdots i \partial^{\mu_{2 i}} \bar{\psi} \overleftrightarrow{i D}^{\mu_{2 i+1}} \cdots i \stackrel{\leftrightarrow}{D}{ }^{\mu_{n-1}} \sigma^{\mu_{n} \nu} \psi
$$

where all $\mu$ indices are symmetrized and $\stackrel{\leftrightarrow}{D}=[\overrightarrow{i D}-\overleftarrow{i D}] / 2$. Using translational invariance, it is easy to see that,

$$
n_{\mu_{1}} \cdots n_{\mu_{n}} e_{\nu}\left\langle P^{\prime} S^{\prime}\left|\mathcal{O}_{n, 2 i}^{\mu_{1} \cdots \mu_{n} \nu}\right| P S\right\rangle=(2 \xi)^{2 i} \mathcal{F}_{n-2 i} .
$$

As one can see from Eq.(12), the operators $\mathcal{O}_{n, 2 i}^{\mu_{1} \cdots \mu_{n} \nu}$ belonging to same $n$ but different $i$ mix. Taking an appropriate linear combination of these, or equivalently, diagonalizing the mixing matrix, is not difficult. One may establish recursion relations using Eq.(12) to finally arrive at,

$$
\begin{aligned}
\frac{d \tilde{\mathcal{O}}_{n}}{d \ln Q^{2}} & =\frac{\alpha_{s}\left(Q^{2}\right)}{2 \pi} C_{F}\left(\frac{3}{2}-2 S(n)\right) \tilde{\mathcal{O}}_{n} \\
\tilde{\mathcal{O}}_{n} & =\sum_{i=0}^{\left[\frac{n-1}{2}\right]} \frac{(-1)^{i} 2^{n-2 i-1} \Gamma\left(n-i+\frac{1}{2}\right)}{(n-2 i-1) ! i ! \Gamma\left(\frac{3}{2}\right)} \mathcal{O}_{n, 2 i}^{\mu_{1} \cdots \mu_{n} \nu}
\end{aligned}
$$

The coefficients in Eq.(17) are those of the Gegenbauer polynomials, $C_{n-1}^{\frac{3}{2}}(x)$. This can be traced to the fact that these polynomials are essentially the Clebsch-Gordan coefficients which occur in the light-cone expansion of operator products that transform irreducibly under the conformal group [26]. 


\section{B. Evolution of the Gluon Distributions $E_{T}$ and $H_{T}$}

The evolution of the gluon helicity-flip distributions can be studied in the same way as for the quark. For convenience, we define,

$$
\begin{aligned}
& \mathrm{F}\left(x, \xi, Q^{2}\right)=n_{\mu} n_{\nu} e_{(\alpha} e_{\beta)}^{\prime} \\
& \times \int \frac{d \lambda}{2 \pi} e^{i \lambda x}\left\langle P^{\prime} S^{\prime}\left|F^{\mu \alpha}\left(-\frac{1}{2} \lambda n\right) e^{-i g \int_{\lambda / 2}^{-\lambda / 2} d \alpha n \cdot A(\alpha n)} F^{\nu \beta}\left(\frac{1}{2} \lambda n\right)\right| P S\right\rangle .
\end{aligned}
$$

where $e_{\alpha}$ and $e_{\beta}^{\prime}$ are two unit vectors in the transverse directions. We are interested in the change of $\mathrm{F}$ under the change of the momentum cut-off $Q^{2}$. Some representative Feynman diagrams are shown in Fig.2.
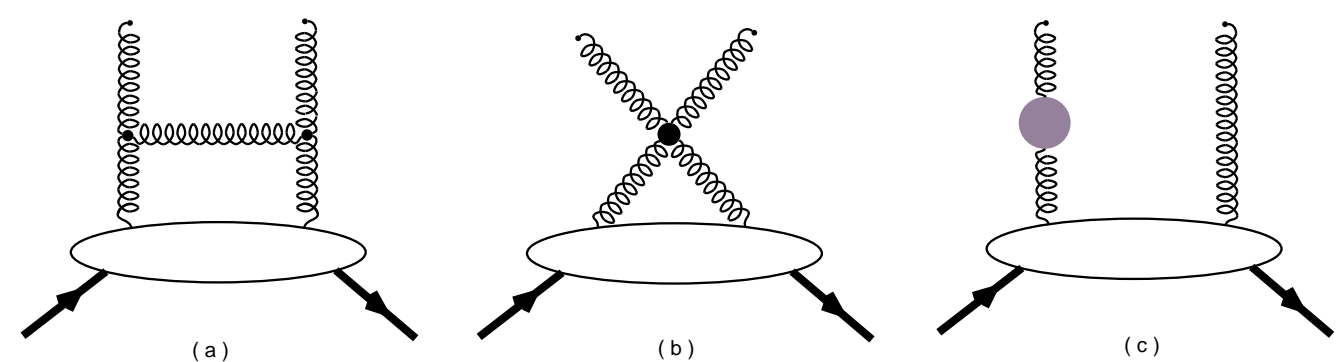

FIG. 2. Representative Feynman diagrams for evolution of gluon helicity-flip parton distributions.

As we have mentioned before, the gluon helicity-flip distributions do not mix with any quark distributions. For $x>\xi$, the evolution equation reads,

$$
\frac{D_{Q} \mathrm{~F}\left(x, \xi, Q^{2}\right)}{D \ln Q^{2}}=\frac{\alpha_{s}\left(Q^{2}\right)}{2 \pi} \int_{x}^{1} \frac{d y}{y} P\left(\frac{x}{y}, \frac{\xi}{y}, \frac{\epsilon}{y}\right) \mathrm{F}\left(y, \xi, Q^{2}\right),
$$

where,

$$
\frac{D_{Q}}{D \ln Q^{2}}=\frac{d}{d \ln Q^{2}}-\frac{\alpha_{s}\left(Q^{2}\right)}{2 \pi} C_{A}\left[\frac{11}{6}-\frac{n_{f}}{3 C_{A}}+\int_{\xi}^{x} \frac{d y}{y-x-i \epsilon}+\int_{-\xi}^{x} \frac{d y}{y-x-i \epsilon}\right],
$$

and,

$$
P(x, y, \epsilon)=2 C_{A} \frac{\left(x^{2}-\xi^{2}\right)}{x\left(1-\xi^{2}\right)^{2}}\left(\frac{2(1-x) \xi^{2}}{x^{2}-\xi^{2}}+\frac{1-\xi^{2}}{1-x+i \epsilon}\right) .
$$

For $-\xi<x<\xi$, the equation is,

$$
\frac{D_{Q} \mathrm{~F}\left(x, \xi, Q^{2}\right)}{D \ln Q^{2}}=\frac{\alpha_{s}\left(Q^{2}\right)}{2 \pi}\left(\int_{x}^{1} \frac{d y}{y} P^{\prime}\left(\frac{x}{y}, \frac{\xi}{y}, \frac{\epsilon}{y}\right)-\int_{-1}^{x} \frac{d y}{y} P^{\prime}\left(\frac{x}{y},-\frac{\xi}{y}, \frac{\epsilon}{y}\right)\right) \mathrm{F}\left(y, \xi, Q^{2}\right),
$$

where,

$$
P^{\prime}(x, \xi, \epsilon)=\frac{\left(x^{2}-\xi^{2}\right)}{x\left(1-\xi^{2}\right)}\left[\frac{2 \xi}{(x-\xi)(1+\xi)}+\frac{4}{1-x+i \epsilon}\right]
$$


And finally for $x<-\xi$, the equation is the same as that for $x>\xi$, except $\int_{x}^{1} \rightarrow-\int_{-1}^{x}$.

In the forward limit, the evolution equation reduces to that for $\Delta(x)$,

$$
\frac{d \Delta(x)}{d \ln Q^{2}}=\frac{\alpha_{s}\left(Q^{2}\right)}{2 \pi} \int_{x}^{1} \frac{d y}{y} P\left(\frac{x}{y}\right) \Delta(y)
$$

where the evolution kernel is,

$$
P(x)=2 C_{A} \frac{x}{(1-x)_{+}}+\left(\frac{11 C_{A}}{6}-\frac{n_{f}}{3}\right) \delta(x-1) .
$$

Here the + prescription is standard [27]. The above result, as far as we know, is new.

It is instructive to look at the evolution in operator form. Define the $n=$ even moments (the odd moments vanish because $\mathrm{F}(x)$ is antisymmetric in $x$ as one can easily check from the definition),

$$
\mathrm{F}_{n}\left(\xi, Q^{2}\right)=\int_{-1}^{1} d x x^{n-1} \mathrm{~F}\left(x, \xi, Q^{2}\right) \quad(n \geq 2)
$$

The evolution equation becomes,

$$
\frac{d \mathrm{~F}_{n}}{d \ln Q^{2}}=\frac{\alpha_{s}\left(Q^{2}\right)}{2 \pi} C_{A}\left[\left(\frac{11}{6}-\frac{n_{f}}{2 C_{A}}-2 S(n)\right) \mathrm{F}_{n}+\sum_{i=1}^{\left[\frac{n-1}{2}\right]}\left(\frac{4 i-2}{n-1}-\frac{4 i+2}{n}+\frac{1}{i}\right)(2 \xi)^{2 i} \mathrm{~F}_{n-2 i}\right] \text {. }
$$

Define a tower of twist-two gluon operators,

$$
\mathcal{O}_{n, 2 i}^{\mu_{1} \cdots \mu_{n} \alpha \beta}=i \partial^{\mu_{1}} \cdots i \partial^{\mu_{2 i}} F^{\mu_{2 i+1} \alpha} \stackrel{\leftrightarrow}{D}{ }^{\mu_{2 i+2}} \cdots i \stackrel{\leftrightarrow}{D}{ }^{\mu_{n-1}} F^{\mu_{n} \beta}
$$

Then it is easy to see,

$$
n_{\mu_{1}} \cdots n_{\mu_{n}} e_{\alpha} e_{\beta}^{\prime}\left\langle P^{\prime} S^{\prime}\left|\mathcal{O}_{n, 2 i}^{\mu_{1} \cdots \mu_{n} \alpha \beta}\right| P S\right\rangle=(2 \xi)^{2 i} \mathrm{~F}_{n-2 i}
$$

Hence the mixing of the different moments of the gluon distributions reflects the mixing of the twist-two operators of same spin and dimension. Define a new basis of operators in term of the Gegenbauer polynomials $C_{n-2}^{\frac{5}{2}}(x)$ combinations,

$$
\mathcal{O}_{n}=\sum_{i=0}^{\left[\frac{n-2}{2}\right]} \frac{(-1)^{i} 2^{n-2 i-2} \Gamma\left(n-i+\frac{1}{2}\right)}{(n-2 i-2) ! i ! \Gamma\left(\frac{5}{2}\right)} \mathcal{O}_{n, 2 i}^{\mu_{1} \cdots \mu_{n} \alpha \beta}
$$

Then the evolution of $\mathcal{O}_{n}$ becomes diagonal,

$$
\frac{d \mathcal{O}_{n}}{d \ln Q^{2}}=\frac{\alpha_{s}\left(Q^{2}\right)}{2 \pi} C_{A}\left(\frac{11}{6}-\frac{n_{f}}{3 C_{F}}-2 S(n)\right) \mathcal{O}_{n}
$$

Again, the above simplification is due to conformal symmetry. However, beyond the leadinglogarithmic order, the conformal symmetry is anomalously broken by quantum corrections 28 . 


\section{PHOTON HELICITY-FLIP COMPTON AMPLITUDE}

The gluon helicity-flip distributions, $H_{T g}(x, \xi)$ and $E_{T g}(x, \xi)$, are basic properties of the nucleon, at par with the other distributions, namely, $H_{g}(x, \xi), E_{g}(x, \xi), \tilde{H}_{g}(x, \xi)$, and $\tilde{E}_{g}(x, \xi)$. Because of angular momentum conservation, the gluon double-helicity flip distributions do not mix with quark distributions, making their isolation and possible measurement relatively cleaner. One would like to know which hard processes probe $H_{T g}$ and $E_{T g}$. The general Compton scattering involving two photons offers one possibility, perhaps the simplest. Diffractive vector meson production from a deeply-virtual photon is another [19.24.

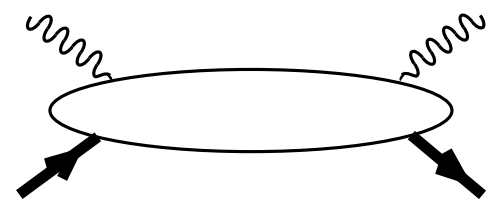

(a)

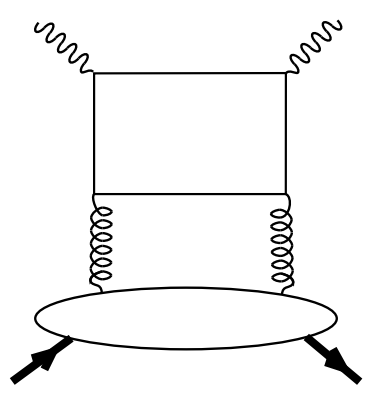

(b)

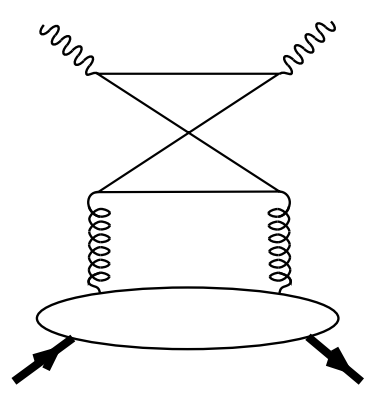

(c)

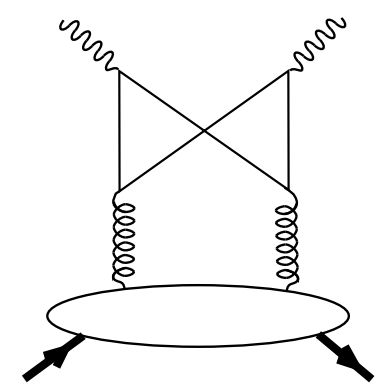

(d)

FIG. 3. Diagrams for photon helicity-flip Compton scattering.

Fig. 3a illustrates the general two photon process. A highly virtual photon with momentum $q+\Delta / 2$ and transverse polarization is incident upon the nucleon. A second photon of momentum $q-\Delta / 2$ is detected, and the recoiling nucleon emerges intact. The scattering amplitude is,

$$
T^{\mu \nu}=i \int d^{4} z e^{-i q \cdot z}\left\langle P^{\prime} S^{\prime}\left|T\left[J^{\nu}\left(-\frac{1}{2} z\right) J^{\mu}\left(\frac{1}{2} z\right)\right]\right| P S\right\rangle
$$

A convenient set of kinematic variables is obtained by choosing $q^{\mu}$ and $\bar{P}^{\mu}=\frac{1}{2}\left(P+P^{\prime}\right)^{\mu}$ to be collinear and in the 3 direction. In terms of $p^{\mu}, n^{\mu}$, we expand the "average" photon momentum,

$$
q^{\mu}=-x_{B} p^{\mu}+\left(Q^{2} / 2 x_{B}\right) n^{\mu}
$$

The transverse components of $\Delta^{\mu}$ are assumed to be of the order of the nucleon mass, i.e. much smaller than the hard momentum $Q$. In the collinear approximation, the momenta of the incoming and outgoing photons are, respectively, $q-\xi p$ and $q+\xi p$. The initial and final state nucleon's momenta are $(1+\xi) p^{\mu}$ and $(1-\xi) p^{\mu}$, respectively. Symmetrizing the diagrams in this manner makes it possible to get simpler expressions by exploiting crossing symmetry. 
There is no tree-level coupling between the scattering photon and the gluons in the target. However, there is a contribution at $O\left(\alpha_{s}\right)$ from the box-diagram and its permutations, shown in Figs. 3b-d. The momenta of the gluons going outward and returning to the nucleon blob are, respectively, $(x+\xi) p^{\mu}$ and $(x-\xi) p^{\mu}$ with $-1<x<1$. The loop momentum is expanded as,

$$
k^{\mu}=(k \cdot n) p^{\mu}+(k \cdot p) n^{\mu}+k_{\perp}^{\mu} .
$$

Rather than use Feynman parameters to solve the integral with four products in the denominator, we systematically exploit crossing symmetry $(\xi \rightarrow-\xi)$ to reduce the integral to a much more manageable form with three product terms. Dimensional regularization is used for the divergent graphs. The infrared singularity cancels in the sum of the three diagrams, as it must. Many details of the calculation are similar to those in Ref. [29].

Our final result for the photon double helicity-flip amplitude is,

$$
T^{\mu \nu}=\frac{\alpha_{s}}{2 \pi}\left(\sum_{q} e_{q}^{2}\right) \int_{-1}^{1} d x \frac{x}{x^{2}-\xi^{2}}\left[1+\frac{x_{B}^{2}-\xi^{2}}{x^{2}-\xi^{2}} \ln \left(\frac{x_{B}^{2}-x^{2}}{x_{B}^{2}-\xi^{2}}\right)\right] n_{\alpha} n_{\beta} \mathcal{T}^{\mu \nu \alpha \beta},
$$

where,

$$
\mathcal{T}^{\mu \nu \alpha \beta}=\frac{1}{x} \int \frac{d \lambda}{2 \pi} e^{i \lambda x}\left\langle P^{\prime} S^{\prime}\left|F^{(\mu \alpha}\left(-\frac{\lambda}{2} n\right) F^{\nu \beta)}\left(\frac{\lambda}{2} n\right)\right| P S\right\rangle .
$$

In the above, $q$ sums over the quarks circulating in the loop and $e_{q}$ are their electric charges. Eq.(35) is the convolution of a perturbatively calculable part and a soft part reflecting the nucleon's composition, and is in the form of a factorization theorem at the lowest order of QCD perturbation theory. To completely justify the use of the soft part, one needs to consider all contributing Feynman diagrams with the hard quark loop connecting with arbitrary number of gluons to the nucleon blob. Any choice of gauge may be made of course, but the final result will be gauge independent. It is easiest to work in the light-cone gauge and with simply two physical gluon fields. In covariant gauge it would be necessary to show that these additional gluons are summed up in the path-ordered integral.

As a check on our calculation, we may consider forward scattering $\xi=0$ on a target with spin $J \geq 1$. In the limit of $x_{B} \rightarrow \infty$, the perturbative part in Eq.(35) can be expanded:

$$
\begin{aligned}
& T^{\mu \nu}=-\frac{\alpha_{s}}{2 \pi}\left(\sum_{q} e_{q}^{2}\right) \sum_{n \text { even=2 }}^{\infty} \frac{1}{x_{B}^{n}} \frac{2}{n+2} \int_{-1}^{1} d x x^{n-1} \mathcal{T}^{\mu \nu \alpha \beta} n_{\alpha} n_{\beta} \\
& =-\frac{\alpha_{s}}{2 \pi}\left(\sum_{q} e_{q}^{2}\right) \sum_{n=2}^{\infty} \frac{2}{n+2} \frac{2^{n} q_{1}^{\mu} \cdots q_{n}^{\mu}}{\left(Q^{2}\right)^{n}} \\
& \times\left\langle P S\left|F^{\mu_{1}} i \stackrel{\leftrightarrow}{D}{ }^{\mu_{2}} \cdots i \stackrel{\leftrightarrow}{\mu^{\mu_{n-1}}} F^{\nu \mu_{n}}\right| P S\right\rangle
\end{aligned}
$$

The above coincides with the result in Ref. [18] except for an overall sign. In the general case, we can convert Eq.(35) result into a generalized operator production expansion with derivative operators,

$$
\begin{aligned}
i \int d^{4} z e^{-i q z} J^{\nu}\left(-\frac{z}{2}\right) J^{\mu}\left(\frac{z}{2}\right)= & -\frac{\alpha_{s}}{2 \pi}\left(\sum_{q} e_{q}^{2}\right) \sum_{n \text { even=2 }}^{\infty} \frac{2}{n(n+2)} \frac{2^{n} q_{1}^{\mu} \cdots q_{n}^{\mu}}{\left(Q^{2}\right)^{n}} \\
& \times \sum_{i=0}^{\left[\frac{n-1}{2}\right]}(n-2 i) \mathcal{O}_{\mu_{1} \cdots \mu_{n} \mu \nu}^{n, 2 i}+\ldots
\end{aligned}
$$


where $\mathcal{O}_{\mu_{1} \cdots \mu_{n} \mu \nu}^{n, 2 i}$ is defined in Eq.(28). We would like to emphasize again that only the $\mu \nu$ symmetric and traceless terms are included in the above equation.

\section{DVCS CROSS SECTION WITH GLUON HELICITY-FLIP DISTRIBUTIONS}

The Compton amplitude in the last section can be used to obtain the cross section for deeply virtual Compton scattering. In DVCS, the final photon is real and hence one has the constaint $x_{B}=\xi$. In this section, we calculate the cross section in this special kinematic limit.

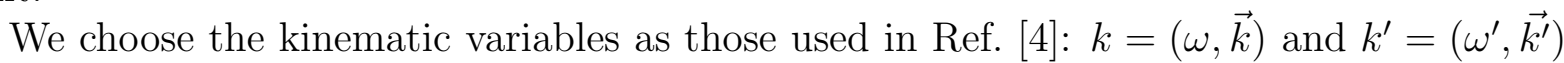
for the four-momenta of the intial and final electrons, $P=(M, 0)$ and $P^{\prime}=\left(E^{\prime}, \vec{P}^{\prime}\right)$ for the initial and final momenta of the nucleon, and $q^{\prime}=\left(\nu^{\prime}, \overrightarrow{q^{\prime}}\right)$ the momentum of the final photon. The differential cross section is,

$$
d \sigma=|\mathcal{T}|^{2} d \Gamma
$$

where $\mathcal{T}$ is the invariant $T$ matrix of the scattering and $d \Gamma$ is the invariant phase space factor. Depending on choice of independent kinematic variables to characterize the differential cross section, $d \Gamma$ takes different form. If one uses the scattered electron's energy and solid angle, and the scattered nucleon's solid angle,

$$
d \Gamma=\frac{1}{32(2 \pi)^{5} \omega M} \omega^{\prime} d \omega^{\prime} d \Omega_{e^{\prime}} d \Omega_{P^{\prime}} \frac{P^{\prime 2}}{\left|P^{\prime}(\nu+M)-q E^{\prime} \cos \phi\right|},
$$

where $\phi$ is the angle between $\vec{q}$ and $\vec{P}^{\prime}$, and the sum over two possible solutions of $\left|\overrightarrow{P^{\prime}}\right|$ is implicit. On the other hand, one can also use the standard $Q^{2}$ and $x_{B}$ (or $s$ ), $t=\Delta^{2}$, the $t$-channel momentum transfer, and $\phi$ the angle between lepton and hadron planes [21, 30].

The invariant $T$ matrix consists of two parts. The first part comes from the Compton scattering,

$$
\mathcal{T}_{1}=-e^{3} \bar{u}\left(k^{\prime}\right) \gamma^{\mu} u(k) \frac{1}{q^{2}} T_{\mu \nu} \epsilon^{\nu *}
$$

where $\bar{u}, u$ are the spinors of the lepton and $\epsilon$ is the polarization of the emitting photon. The Compton amplitude $T^{\mu \nu}$ contains both photon helicity-flip and non-flip contributions,

$$
T_{\mu \nu}=T_{\mu \nu}^{\Delta \lambda=0}+T_{\mu \nu}^{\Delta \lambda=2}
$$

where the first term is given by Eq.(4) in Ref. [4] and the second term is from Eq.(35),

$$
\begin{aligned}
T_{\Delta \lambda=2}^{\mu \nu}= & \frac{\alpha_{s}}{4 \pi}\left(\sum_{i} e_{i}^{2}\right) \int_{-1}^{1} d x\left(\frac{1}{x-\xi+i \epsilon}+\frac{1}{x+\xi-i \epsilon}\right) \\
& \times n_{\alpha} n_{\beta}\left[H_{T g}(x, \xi) \bar{U}\left(P^{\prime} S^{\prime}\right) \frac{\bar{P}^{([\mu} i \Delta_{\perp}^{\alpha]} \sigma^{\nu \beta)}}{M} U(P S)\right. \\
& \left.+E_{T g}(x, \xi) \bar{U}\left(P^{\prime} S^{\prime}\right) \frac{\bar{P}^{([\mu} \Delta_{\perp}^{\alpha]}}{M} \frac{\left.\gamma^{[\nu} \Delta_{\perp}^{\beta]}\right)}{M} U(P S)\right]
\end{aligned}
$$




$$
\begin{aligned}
= & \frac{\alpha_{s}}{4 \pi}\left(\sum_{q} e_{q}^{2}\right) \int_{-1}^{1} d x\left(\frac{1}{x-\xi+i \epsilon}+\frac{1}{x+\xi-i \epsilon}\right) \frac{1}{4 M} \\
& \times\left[H_{T g}(x, \xi) \bar{U}\left(P^{\prime}\right)\left(\Delta_{\perp}^{\mu} \gamma_{\perp}^{\nu}+\Delta_{\perp}^{\nu} \gamma_{\perp}^{\mu}-g_{\perp}^{\mu \nu} \Delta_{\perp}\right) \not h U(P)\right. \\
& \left.+\frac{1}{M} E_{T g}(x, \xi) \bar{U}\left(P^{\prime}\right)\left(\xi\left(\Delta_{\perp}^{\mu} \gamma_{\perp}^{\nu}+\Delta_{\perp}^{\nu} \gamma_{\perp}^{\mu}-g_{\perp}^{\mu \nu} \Delta_{\perp}\right)+\not h\left(\Delta_{\perp}^{\mu} \Delta_{\perp}^{\nu}-\frac{1}{2} g^{\mu \nu} \Delta_{\perp}^{2}\right)\right) U(P)\right] .
\end{aligned}
$$

The second part of the $\mathrm{T}$ matrix comes from the Bethe-Heitler process,

$$
\mathcal{T}_{2}=-e^{3} \bar{u}\left(k^{\prime}\right)\left[\nexists^{*} \frac{1}{\not k-\not \Delta-m_{e}+i \epsilon} \gamma^{\mu}+\gamma^{\mu} \frac{1}{\not k^{\prime}+\not \Delta-m_{e}+i \epsilon} \phi^{*}\right] u(k) \frac{1}{\Delta^{2}}\left\langle P^{\prime}\left|J_{\mu}(0)\right| P\right\rangle,
$$

where $m_{e}$ is the mass of electron and will be ignored for the following discussion. The elastic nucleon matrix element is,

$$
\left\langle P^{\prime}\left|J_{\mu}(0)\right| P\right\rangle=\bar{U}\left(P^{\prime}\right)\left[\gamma_{\mu} F_{1}\left(\Delta^{2}\right)+F_{2}\left(\Delta^{2}\right) \frac{i \sigma_{\mu \nu} \Delta^{\nu}}{2 M}\right] U(P)
$$

where $\bar{U}, U$ are the nucleon spinors and $F_{1}$ and $F_{2}$ are the usual Dirac and Pauli form factors of the nucleon.

We are interested in only the leading contribution to the cross section from the helicityflip gluon distributions. This comes from the interferences between the helicity-flip and non-flip Compton amplitudes and between the former and the Bethe-Heitler amplitude. The first interference yields,

$$
\left(\mathcal{T}_{1}^{\Delta \lambda=0}\right)^{*} \mathcal{T}_{1}^{\Delta \lambda=2}+\mathcal{T}_{1}^{\Delta \lambda=0}\left(\mathcal{T}_{1}^{\Delta \lambda=2}\right)^{*}=-\frac{e^{6}}{Q^{4}} \ell_{\mathrm{VC}}^{\mu \nu} W_{\mathrm{VC} \mu \nu}
$$

where the lepton tensor $\ell_{\mathrm{VC}}^{\mu \nu}$ can be found in Ref. 四. The hadron tensor is

$$
\begin{aligned}
W_{\mathrm{VC}}^{\mu \nu}= & \frac{1}{M^{2}}\left(\Delta_{\perp}^{\mu} \Delta_{\perp}^{\nu}-\frac{1}{2} g_{\perp}^{\mu \nu} \Delta_{\perp}^{2}\right) \frac{\alpha_{s}}{4 \pi}\left(\sum_{q} e_{q}^{2}\right) \\
& \times \sum_{q} e_{q}^{2} \operatorname{Re} \int_{-1}^{1} d x \alpha(x) \int_{-1}^{1} d x^{\prime} \alpha^{*}\left(x^{\prime}\right)\left[H_{q}(x, \xi) E_{T g}\left(x^{\prime}, \xi\right)-H_{T g}\left(x^{\prime}, \xi\right) E_{q}(x, \xi)\right] .
\end{aligned}
$$

The tensor structure $\left(\Delta_{\perp}^{\mu} \Delta_{\perp}^{\nu}-\frac{1}{2} g^{\mu \nu} \Delta_{\perp}^{2}\right)$ signals a $\cos 2 \phi$ term in the cross section, as was noted in 21. There is, of course, also a gluon helicity non-flip term but it enters at one higher power of $\alpha_{s}$.

The interference between the double-helicity-flip Compton and Bethe-Heitler amplitudes is,

$$
\mathcal{T}_{1}^{\Delta \lambda=2^{*}} \mathcal{T}_{2}+\mathcal{T}_{1}^{\Delta \lambda=2} \mathcal{T}_{2}^{*}=2 \frac{e^{6}}{\Delta^{2} Q^{2}} \ell^{(\mu \nu) \alpha} \operatorname{Re} H_{(\mu \nu) \alpha}
$$

where $\ell^{(\mu \nu) \alpha}$ depends on electron kinematic variables and can be found in [4]. The nucleon structure dependent part is, 


$$
\begin{aligned}
H^{(\mu \nu) \alpha}= & \left(\Delta_{\perp}^{\mu} \Delta_{\perp}^{\nu}-\frac{1}{2} g_{\perp}^{\mu \nu} \Delta_{\perp}^{2}\right) \frac{\alpha_{s}}{4 \pi}\left(\sum_{q} e_{q}^{2}\right) \int_{-1}^{1} d x \alpha(x) \\
& \times\left[\left(F_{1}+F_{2}\right)\left(H_{T g}(x, \xi)+\frac{\Delta^{2}}{4 M^{2}} E_{T g}(x, \xi)\right) n^{\alpha}+\left(F_{1} E_{T g}(x, \xi)-F_{2} H_{T g}(x, \xi)\right) \frac{\bar{P}^{\alpha}}{M^{2}}\right] \\
& +\left(\Delta_{\perp}^{\mu} g_{\perp}^{\alpha \nu}+\Delta_{\perp}^{\nu} g_{\perp}^{\alpha \mu}-g_{\perp}^{\mu \nu} \Delta_{\perp}^{\alpha}\right) \frac{\alpha_{s}}{4 \pi}\left(\sum_{q} e_{q}^{2}\right) \int_{-1}^{1} d x \alpha(x) \\
& \times \xi\left(F_{1}+F_{2}\right)\left(H_{T g}(x, \xi)+\frac{\Delta^{2}}{4 M^{2}} E_{T g}(x, \xi)\right) .
\end{aligned}
$$

Here the presence of $\Delta$ in $\ell^{\mu \nu \alpha}$ and $\Delta^{\mu} \Delta^{\nu}$ in $H^{(\mu \nu) \alpha}$ can give rise to a distinct $\cos 3 \phi$ terms in the cross section [21]. To obtain the latter, one just multiplies the lepton and hadron tensors together to get the square of the $T$ matrix. Because of its length, we omit the final expression.

A more direct way to see the angular dependence of the cross section is to use the formulas derived in the center-of-mass frame in Refs. [21,30]. According to these works, all one needs is the hadron helicity amplitude $M_{H, H^{\prime}}^{\lambda, \lambda^{\prime}}$, where $\lambda, \lambda^{\prime}$ and $H, H^{\prime}$ are the initial and final photon and nucleon helicities, respectively. The helicity-flip nucleon amplitude $M_{H, H^{\prime}}^{-1,1}$ clearly is just the helicity-flip Compton amplitude,

$$
M_{h, h^{\prime}}^{-1,1}=\epsilon_{\alpha}(-1) \epsilon_{\beta}^{*}(+1) T_{\Delta \lambda=2}^{\alpha \beta} .
$$

This can readily be evaluated using Eq.(43) by substituting in the appropriate Dirac spinor for the nucleon helicity states. According to [21], certain angular weighted cross sections can be used to make a direct extraction of the above amplitude.

\section{SUMMARY AND COMMENTS}

In this paper, we have presented a number of new results related to the helicity-flip off-forward parton distributions. First, we enumerated systematically all leading-twist offforward parton distributions for a nucleon: six for the quark parton and another six for the gluon. Four of these distributions, two each for the quark and gluon partons, involve parton helicity-flip. Second, we derived the leading-logarithmic evolution equations for these helicity-flip disitributions. In the forward limit, our result agrees with the known kernel for $h_{1}(x)$ while the kernel for $\Delta(x)$ is new. Third, we obtained the photon helicity-flip Compton amplitude in terms of a tower of gluon operators with total derivatives. Our result may be obtained from the known forward case by using the conformal symmetry of QCD. Finally, we compute the leading DVCS cross section which depends on the gluon helicity-flip distributions.

We have emphasized the unique role played by helicity-flip distributions in characterizing the properties of the nucleon. If one askes for a clear experimental signal of existence of gluons in the nucleon, the helicity-flip Compton amplitude would serve the purpose. Without the vector gluons, it would be at least power suppressed in the high-energy limit. Of course, the helicity-flip gluon distributions can also be measured in vector meson production [19]. The size of the helicity-flip distributions should be similar to the usual helicity-dependent parton distributions - there is no extra suppression in the soft physics to curb helicity flip. 


\section{ACKNOWLEDGMENTS}

We would like thank J. Osborne for discussions and for drawing the Feynman diagrams. PH thanks the Fulbright Foundation for sponsoring his visit to the University of Maryland. This work is supported in part by funds provided by the U.S. Department of Energy (D.O.E.) under cooperative agreement DOE-FG02-93ER-40762. 


\section{REFERENCES}

[1] K. Watanabe, Prog. Theo. Phys. 67, 1834 (1982);

F. M. Dittes et. al., Phys. Lett. B 209 (1988) 325;

D. Müller et. al., Fortschr. Phys. 42 (1994) 101.

[2] X. Ji, Phys. Rev. Lett. 78, 610 (1997).

[3] A. V. Radyushkin, Phys. Lett. B380, 417 (1996); Phys. Lett. B385 (1996) 333; Phys. Rev. D56, 5524, 1997.

[4] X. Ji, Phys. Rev. D55, 7114 (1997).

[5] P. Hoodbhoy, Phys. Rev. D56, 388, 1997.

[6] I. I. Balitsky and A. V. Radyushkin, Phys. Lett. B413, 114 (1997).

[7] J. Blümlein, B. Geyer, and D. Robaschik, Phys. Lett. B406, 161 (1997).

[8] X. Ji, W. Melnitchouk, and X. Song, Phys. Rev. D56, 5511 (1997).

[9] L. L. Frankfurt, A. Freund, V. Guzey, and M. Strikman, hep-ph/9703449.

[10] V. Yu. Petrov et al., hep-ph/9710270.

[11] A. V. Belitsky and D. Müller, hep-ph9709379.

[12] A. V. Belitsky, B. Geyer, D. Müller, and A. Schäfer, hep-ph/9710427.

[13] L. Mankiewicz, G. Piller, E. Stein, M. Vanttinen, T. Weigl, hep-ph/9712251.

[14] J. Ralston and D. Soper, Nucl. Phys. B152, 109 (1979).

[15] A. V. Belitsky and D. Muller, hep/ph9709379.

[16] X. Artru and M. Mekhfi, Z. Phys. C45, 669 (1990).

[17] R. L. Jaffe and X. Ji, Phys. Rev. Lett. 67 (1991) 552.

[18] R.L.Jaffe and A.V.Manohar, Phys. Lett. B223, 218 (1989).

[19] J. C. Collins, L. Frankfurt, and M. Strikman, Phys. Rev. D56, 2982 (1997).

[20] L. Mankiewicz, G. Piller, and T. Wiegl, hep-ph/9711227.

[21] M. Diehl, T. Gousset, B. Pire, and J. P. Ralston, Phys. Lett. B411, 193 (1997).

[22] R. L. Jaffe, Nucl. Phys. B229 (1983) 205.

[23] M. Diehl and T. Gousset, DAPNIA-SPHN-98-01, e-Print Archive: hep-ph/9801233.

[24] S. J. Brodsky et al., Phys. Rev. D50, 3134 (1994).

[25] M. Nzar and P. Hoodbhoy, Phys.Rev. D45, 2264 (1992).

[26] S. Ferrara, R. Gatto, and A. F. Grillo, Nucl. Phys. B34, 349 (1971).

Th. Ohrndorf, Nucl. Phys. B198, 26 (1982).

[27] G. Altarelli and G. Parisi, Nucl. Phys. B126, 298 (1977).

[28] D. Müller, hep-ph/9704406.

[29] X. Ji and J. Osborne, UMD PP\#98-074, hep-ph/9801260.

[30] P. Kroll, M. Schurmann, and P. A. M. Guichon, Nucl. Phys. A598, 435 (1996). 